NBER WORKING PAPER SERIES

\title{
INEQUALITY CHANGE IN CHINA AND \\ (HUKOU) LABOUR MOBILITY RESTRICTIONS
}

John Whalley

Shunming Zhang

Working Paper 10683

http://www.nber.org/papers/w10683

\section{NATIONAL BUREAU OF ECONOMIC RESEARCH 1050 Massachusetts Avenue Cambridge, MA 02138}

August 2004

We thank Jim Davies, Stephen McGurk, Randy Spence and Terry Sicular and to seminar groups at the University of Western Ontario for their comments. We are grateful for support under the IDRC - MIMAP programme. We also acknowledge helpful comments from a seminar group at a MIMAP meeting in Manila (February 2003). The views expressed herein are those of the author(s) and not necessarily those of the National Bureau of Economic Research.

(C)2004 by John Whalley and Shunming Zhang. All rights reserved. Short sections of text, not to exceed two paragraphs, may be quoted without explicit permission provided that full credit, including (C) notice, is given to the source. 
Inequality Change in China and (Hukou) Labour Mobility Restrictions

John Whalley and Shunming Zhang

NBER Working Paper No. 10683

August 2004

JEL No. J61, R23, F00

\section{ABSTRACT}

We analyze the Hukou system of permanent registration in China which many believe has supported growing relative inequality over the last 20 years by restraining labour migration both between the countryside and urban areas and between regions and cities. Our aim is to inject economic modelling into the debate on sources of inequality in China which thus far has been largely statistical. We first use a model with homogeneous labour in which wage inequality across various geographical divides in China is supported solely by quantity based migration restrictions (urban -- rural areas, rich -poor regions, eastern coastal -- central and western (noncoastal) zones, eastern and central -- western development zones, eastern -- central -- western zones, more disaggregated 6 regional classifications, and an all 31 provincal classification). We calibrate this model to base case data and when we remove migration restrictions all wage and most income inequality disappears. Results from this model structure point to a significant role for Hukou restrictions in supporting inequality in China, and show how economic rather than statistical modelling can be used to decompose inequality change. We then modify the model to capture labour efficiency differences across regions, calibrating the modified model to estimates of both national and regional Gini coefficients. Removal of migration barriers is again inequality improving but now less so. Finally, we present a further model extension in which urban house price rises retard rural - urban migration. The impacts of removing of migration restrictions on inequality are smaller, but are still significant.

John Whalley

Department of Economics

Social Science Centre

University of Western Ontario

London, Ontario N6A 5C2 CANADA

and NBER

jwhalley@uwo.ca
Shunming Zhang

University of Western Ontario

szhang@uwo.ca 


\section{INTRODUCTION}

The statistical literature on inequality in China (Bramall and Jones (1993), Chen (1996), Hare and West (1999), Jalan and Ravallion (1998), Kanbur and Zhang (1999), Lyons (1991), Rozelle (1994), and Tsui (1991, $1993,1996,1998,1998))$ is widely interpreted as pointing to growing national relative inequality in recent years. According to received wisdom based on some of this literature, the national Gini coefficient from China on income inequality has increased to around 0.4 from 0.3 over the last 10 years. ${ }^{2}$ This change coexists with more slowly growing inequality within the urban and rural segments of the economy (as measured by Gini coefficients), and also within coastal zones and inland segments of the economy. A sharp increase in the income / capita gap across these divides is usually taken to account for increased national relative inequality. Absolute poverty as measured by head count ratios and other measures consistently falls in Chinese data reflecting strong GDP growth in recent years. Numerous attempts have been made to account for this inequality change profile using statistical techniques as in the literature listed above. Our aim here is to use economic modelling to provide fresh decompositional insights as to how much of the recent growth in inequality in China is due to which factors.

We focus on the system of Hukou in China, or registered permanent residence, which is location specific. Not having Hukou in urban areas means that migrants receive no education or health benefits and cannot purchase housing, since title to it cannot be registered by them. Effectively, Hukou operates as a barrier to urban / rural migration in China and supports large regional wage differentials which labour markets do not compete away. We ask how much inequality there would have been in China without the Hukou system, and consciously try to introduce results from the application of numerical modelling techniques into a debate which has been dominated by detailed data analysis and speculation rather than model based counterfactual analysis.

Our starting point is the literature on the global consequences of immigration restrictions (see Hamilton and Whalley (1984)). In this literature, differences in both wage rates and GDP / capita across countries are assumed to be supported by immigration restrictions in a world with country specific factor inputs and downward sloping marginal product of labour schedules for otherwise potentially mobile labour. Parameters for an assumed underlying technology are calibrated so as to be consistent with observed data on wage differentials, labour shares of income, and GDP and population by country, and counterfactuals performed to analyze the impacts of immigration barrier removal. Assumptions that there is homogeneous labour across countries, or that there are efficiency differences across countries are used as alternatives in these exercises.

Here we make calculations for China as to what the impacts of removing internal (Hukou) barriers to regional labour mobility on inequality could be using data on both aggregate and regional GDP / capita using similar methods. In so doing we elaborate on the earlier methodology used to analyze global migration restrictions by using a simple basic model for which we sequentially develop a number of model elaborations of increasing complexity. We first ignore inequality within regions and treat labour as homogeneous both

\footnotetext{
${ }^{2}$ We note that there is suprising disagreement even over this central alleged fact regarding inequality trends in China (see Chang (2002) and Zhang, Liu and Yao (2001)). Chang (2002) reports results of a number of studies in which data on GDP or income / capita across provinces in China show only small changes in relative inequality over time, and even reversals (in contrast to current received wisdom). It is seemingly only in survey data and particularly in the World Bank Living Standards Surveys that large changes in income inequality in China of approximately the degree suggest above are reported.
} 
across regions and across individuals. In this model wage rates across regions are equalized when migration restrictions are removed. With region specific fixed factors, regional differences in GDP / capita do not disappear with barrier removal although they fall sharply and national inequality is much reduced. Significant efficiency gains also accrue from barrier removal in this model.

We then extend the basic model by also assuming a distribution of efficiency types within each region and calibrating an extended model to both regional and national estimates of Gini coefficients before barrier removal, and also regional and national data on GDP / capita. We again remove migration barriers and recalculate national inequality in the absence of Hukou restrictions. Significant efficiency impacts again occur but reductions in national inequality are smaller.

In a second elaboration we introduce region specific house price effects and capture their dampening impacts on migration. This is motivated by the desire to also capture the impacts of sharp increases in urban house prices and housing rents in China on urban - rural mobility over the last 10 years. We develop a two good general equilibrium model with goods and housing, in which location specific housing stocks support differing urban and rural house prices. Equilibrium migration conditions equalize the real value of wage rates and we incorporate differences in house prices across regions through region specific true cost of living indices. Removing Hukou restrictions in this model again generates labour flows into urban areas which now drive up urban house prices which in turn dampens migration. Labour flows under Hukou removal are smaller and efficiency gains are smaller, but significant redistribution occurs between urban dwellers whose house prices rise and rural dwellers whose house prices fall.

While findings from these exercises are data and parameter sensitive, they nonetheless jointly point to significiant impacts from the Hukou system in supporting growing overall relative inequality in China, and significant efficiency gains from its removal. They also show how economic modelling as well as statistical techniques can be used to decompose inequality change into various components not only for China but also for other countries. 


\section{SIMPLE INEQUALITY DECOMPOSITIONS USING A BASIC MODEL}

The objective of our paper is to assess how inequality in China might behave were it not for the Hukou system of permanent registration acting as a set of restrictions on labour mobility. We calibrating models of inter-regional labour mobility in China to base case data incorporating both national and regional Gini coefficient estimates in the presence of Hukou restrictions and eliminate these restrictions in the model and recalculate national and regional Gini coefficients as the model counterfactual. We use the same techniques to assess how many people might migrate, and what the economy wide efficiency gains might be.

We first use a simple model in which each region produces a single good $Y$ according to a region specific production function in which labour enters as a homogenous factor input which is mobile across all regions. With decreasing returns to scale production there are returns to region specific fixed factors (rents) and so in the fully mobile labour case even if wage rates are equalized across regions, GDP per capita across regions will not be.

We exposit the model structure algebraically for the general case of $S$ regions. If we assume $S=2$ we can group China data in a number of ways: urban and rural; rich and poor; eastern coastal and central and western (non-coastal) zones; eastern and central and western development zones. If $S=3$, we group by eastern, central and western zones. We also consider a 6 region case of Northern China, Northeastern China, Eastern China, Central and Southern China, Southwestern China, and Northwestern China. There are 31 provinces, centrally administered municipalities and autonomous regions in China in total ${ }^{3}$ and we also explore a 31 region variant of the model that captures all of these. Differences in results caross these cases reflect the degree and form of regional disaggregation in empirical application of the model.

In the model,

$$
Y_{s}=f_{s}\left(L_{s}\right), \quad s=1, \cdots, S
$$

where $Y_{s}$ is production in region $s, L_{s}$ is labour used in region $s, f_{s}^{\prime}>0$ and $f_{s}^{\prime \prime}<0$, and we assume that $\sum_{s} L_{s}=L$ (full employment). Labour market clearing across regions determines the common wage $W=W_{s}$ $(s=1, \cdots, S)$.

Where there are no barriers to labour mobility, i.e., labour receives its marginal product in all regions, i.e.

$$
W=f_{s}^{\prime}\left(L_{s}\right), \quad s=1, \cdots, S .
$$

If there are barriers to mobility of labour across regions, then the interregional labour allocation no longer corresponds to that supporting a market clearing wage. Suppose this allocation is given as $L_{s}^{0}(s=1, \cdots, S)$ such that $\sum_{s} L_{s}=L$, then the marginal products of labour across regions will differ and wage rates $W_{s}$ $(s=1, \cdots, S)$ will also differ, i.e. $f_{s}^{\prime}\left(L_{s}^{0}\right)$ will differ across $s$. Removing interregional barriers to labour mobility in this case implies an efficiency gain for the economy if marginal products of labour are the equalized across regions.

Figure 1 illustates the case of a 2 region urban - rural economy. Here, initial migration restrictions support wage rate differences across regions while the common wage $W=W_{U}=W_{R}$ applies when migration restrictions are removed. Income per capita across the two regions still differs with no migration restrictions

\footnotetext{
${ }^{3}$ We treat all the provinces, centrally administered municipalities and autonomous regions in China as separate and distinct entitles (provinces) in the detailed regional calculations reported on later.
} 
as region specific rents differ. Income inequality is much reduced but not eliminated in this case as wage rates converge across regions. An efficiency gain accrues, as shown, to the whole economy. 
Figure 1. Gains from Eliminating Labour Mobility Restrictions in a 2 Region Model

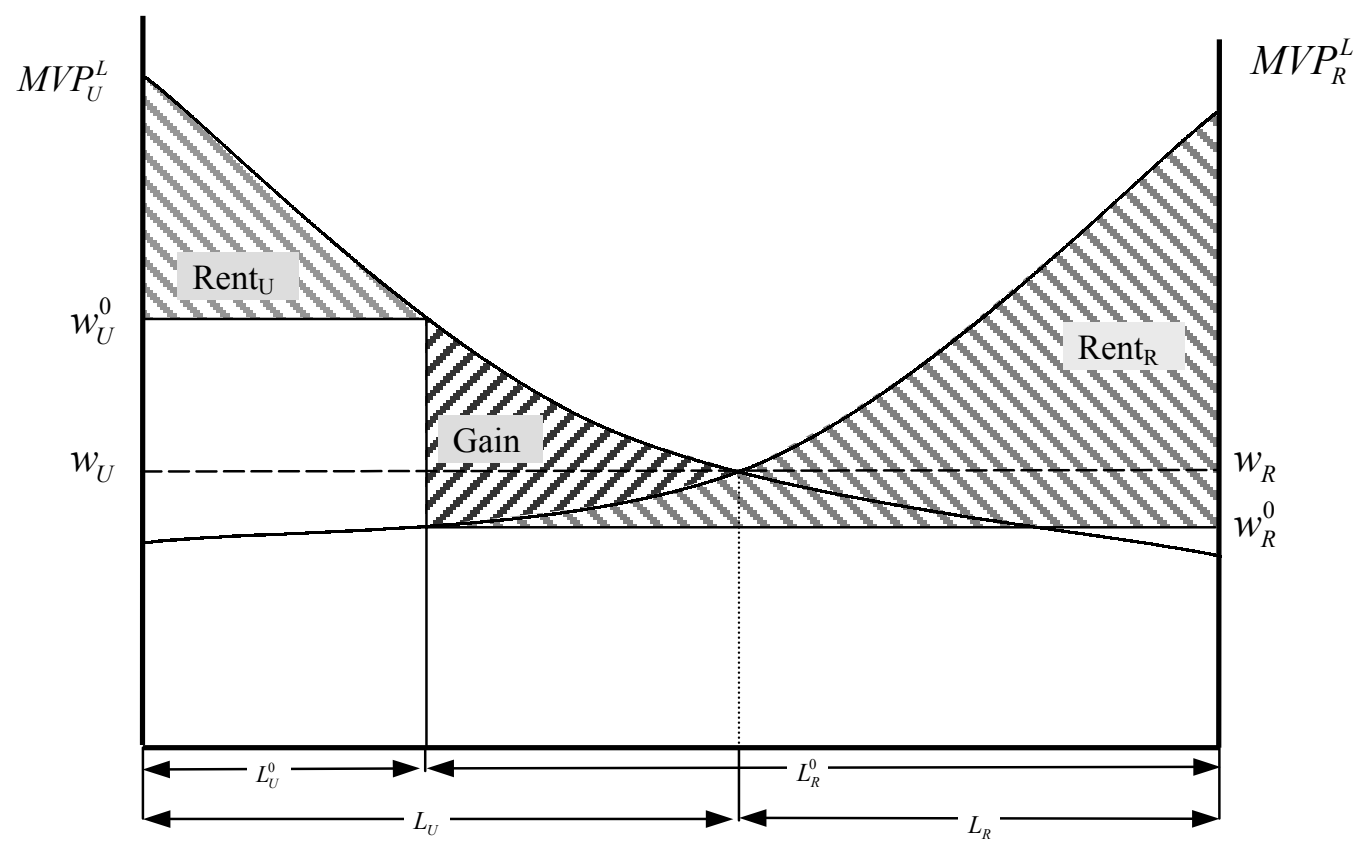


If we assume a diminishing marginal product production function in each region of the form

$$
Y_{s}=A_{s} L_{s}^{\alpha_{s}}, \quad s=1, \cdots, S
$$

then with decreasing marginal productivity of labour the regional wage $W_{s}$ is given as

$$
W_{s}=\frac{\partial Y_{s}}{\partial L_{s}}=\alpha_{s} A_{s} L_{s}^{\alpha_{s}-1}, \quad s=1, \cdots, S
$$

and regional rents, $R_{s}$, are ${ }^{4}$

$$
R_{s}=Y_{s}-W_{s} L_{s}, \quad s=1, \cdots, S .
$$

Income in region $s, I_{s}$, is given by

$$
I_{s}=Y_{s}, \quad s=1, \cdots, S .
$$

and, assuming equal proportional shares in rents with the region, income per worker in each region, $J_{s}$, is given by the average product of labour $J_{s}=\frac{I_{s}}{L_{s}}=\frac{Y_{s}}{L_{s}}, s=1, \cdots, S$.

In equilibrium

$$
\sum_{s} L_{s}=L
$$

where $L$ is the national endowment of labour.

Typically, the size of the work force and the population by regional will differ. If the total national population is $N$, and the population in region $s$ is $N_{s}$, then $N=\sum_{s} N_{s}$, and the average income in each region $s, \bar{I}_{s}$, is given by

$$
\bar{I}_{s}=\frac{I_{s}}{N_{s}}=\frac{Y_{s}}{N_{s}}=\frac{R_{s}}{N_{s}}+\frac{W_{s} L_{s}}{N_{s}}, \quad s=1, \cdots, S
$$

National income $I=\sum_{s} I_{s}=\sum_{s} Y_{s}$, and national average income, $\bar{I}$, is

$$
\bar{I}=\frac{I}{N}=\frac{\sum_{s} I_{s}}{\sum_{s} N_{s}}=\frac{\sum_{s} Y_{s}}{\sum_{s} N_{s}} .
$$

The average wage per individual in region $s$ is

$$
\bar{W}_{s}=\frac{W_{s} L_{s}}{N_{s}}, \quad s=1, \cdots, S
$$

in which case $\bar{I}_{s}=\frac{R_{s}}{N_{s}}+\bar{W}_{s}$.

The model set out above can be used numerically to assess the distributional impacts of removing Hukou migration restrictions by calibrating the model to observed wage and GDP / capita differences across the various two region divides set out above for China. To do this we use a benchmark data set for a given initial year in the presence of Hukou restrictions on labour mobility, and assess the implications of removing mobility restrictions given the observed initial differences in GDP / capita and wage inequality. If $Y_{s}, L_{s}$, and $W_{s}$ are observed and given by data, we can use the model in calibration mode and solve the system of $2 S$ equations (3) and (4) for the $2 S$ unknowns $A_{s}$ and $\alpha_{s}$. We can then compute a counterfactual equilibrium for this simple model in which $W=W_{s}(s=1, \cdots, S)$ and labour mobility restrictions are eliminated. Comparing the original data to the solution generated as the counterfactual model equilibrium then yields

\footnotetext{
${ }^{4}$ We assume here that labour migrates only in response to its regional wage, not also its proportional share in rents. The model can be recast in this form and different numerical results will apply.
} 
an evaluation of the impacts of Hukou on both national and regional income inequality in this simple case. This same approach can be used in 2 region on more detailed multi-region calculations.

In Table 1 we report estimates of urban, rural and national household income Gini coefficients from Chang (2002) which show how income inequality has changed China over the last 20 or so years. This is representative of data claimed to show the growing income divide in China (but see the earlier footnote 1 on the implications of alternative data for recent year). At national level there is consistent worsening of national inequality which is seemingly always more unequal than regional inequality (as measured by the Gini coefficient). This is representative of data claimed to show the growing income divide in China (again see footnote 1 over alternative claims for recent years). 
Table 1. Regional and National Income Inequality in China between 1978 and 2000 Gini Coefficients for Household Income

\begin{tabular}{|c|c|c|c|}
\hline Year & Urban Areas & Rural Areas & Nation \\
\hline 1978 & 0.160 & 0.212 & \\
\hline 1979 & 0.160 & 0.241 & \\
\hline 1980 & 0.150 & 0.241 & 0.330 \\
\hline 1981 & 0.150 & 0.232 & \\
\hline 1982 & 0.150 & 0.246 & \\
\hline 1983 & 0.160 & 0.244 & \\
\hline 1984 & 0.190 & 0.227 & 0.300 \\
\hline 1985 & 0.190 & 0.304 & \\
\hline 1986 & 0.200 & 0.305 & \\
\hline 1987 & 0.230 & 0.303 & \\
\hline 1988 & 0.230 & 0.310 & \\
\hline 1989 & 0.230 & 0.310 & \\
\hline 1990 & 0.240 & 0.307 & \\
\hline 1991 & 0.250 & 0.313 & \\
\hline 1992 & 0.270 & 0.329 & \\
\hline 1993 & 0.300 & 0.321 & \\
\hline 1994 & 0.280 & 0.342 & 0.400 \\
\hline 1995 & 0.280 & 0.323 & 0.415 \\
\hline 1996 & 0.290 & 0.329 & 0.424 \\
\hline 1997 & 0.300 & 0.337 & 0.425 \\
\hline 1998 & 0.295 & 0.336 & 0.456 \\
\hline 1999 & & & 0.457 \\
\hline 2000 & 0.320 & & 0.458 \\
\hline
\end{tabular}

Source: Chang (2002)

Tables 2 and 3 report the data we use to calibrate alternative versions of the model. Since data on Chinese GDP / capita (average income) and wage differentials are only readily available on a provincial basis, we use data for all 31 provinces, centrally administered municipalities and autonomous regions to group regions in China in various ways. We consider seven different region divides grouping data on population, output, work force, and wage rate for each.

We first rank provinces from rich to poor (on an income (GDP) / capita basis), taking the top 10 provinces (approximately $35 \%$ of the population) as the rich group. The remaining provinces make up the poor group. We next group provinces into two eastern coastal and central and southern (non-coastal) provincial groupings, and two eastern and central and western development zones. We also divide the 31 provinces into 3 zones and 6 regions. We finally consider all Chinese provinces in a 31 region version of the 
model. 5

In Table 2 and 3 we report the 2001 base year data used to calibrate the 2, 3, and 6 region, and the full 31 regions versions of the labour mobility model. These are GDP / capita by region, wage rate differentials across regions work force and population by region. Using these data as an input, through calibration we determine the model parameters $A_{s}$ and $\alpha_{s}$, as well as regional outputs $Y_{s}^{0}$, rents $R_{s}^{0}$, and $I_{s}^{0}, \bar{I}_{s}^{0}, I^{0}, \bar{I}^{0}$, $J_{s}^{0}, J^{0}$, and $\bar{W}_{s}^{0}$. We assess the impacts of eliminating Hukou restrictions on $Y_{s}, L_{s}, W_{s}, R_{s}, I_{s}, \bar{I}_{s}, I, \bar{I}$, $J_{s}, J, \bar{W}_{s}$, and solving the model in counterfactual model for the case where wage rates are equalized across regions determine the migration impacts on work force and population by region.

Tables 4 and 5 report results for numerical simulations showing the impacts of Hukou elimination in model variants using alternative Chinese regional divides. Table 4 reports cases for four two way divides, two three way divides, and a six region divide. Table 5 reports results from the more detailed 31 province calculation. In each case wage differentials across regional divides are eliminated with the removal of Hukou restrictions, and average incomes across regions are more closely equalized. The differences that remain in average incomes are due to differences in the size of rents across regions.

For the urban-rural 2 region case in Table 4 the per capita income differential falls from $2: 1$ to $7: 10$, while in case two (rich-poor) it falls from over $7: 3$ to $4: 3$. In the urban - rural case, approximately $48 \%$ of the work force and $45 \%$ of the population move from rural to urban areas after Hukou removal. Around $17 \%$ of the population remains in rural areas. They become much richer, their average income (GDP per capita) being 1.42 times higher than that in urban areas. Total output increases by about 13\%, and GDP per capita and income per worker both increase. In the rich - poor case in Table 4, there are smaller migration effects after Hukou removal (approximately $25 \%$ of the work force and the population move), and total output increases only by about $3.2 \%$. In the other 2 region case (EC - CW and EC - WD) there are smaller migration effects from Hukou removal, while total output and GDP per capita and income per worker again increase. In the 3 region E - C - W case there are big migration effects between Central - Western and Eastern zones after Hukou removal. Total output increases by about 3.0\%. In the 6 region case, migration effects between regions are small, and total output increases by about $1.7 \%$.

\footnotetext{
${ }^{5}$ There are 31 provinces, centrally administered municipalities and autonomous regions in the Chinese mainland. The 31 provinces, centrally administered municipalities and autonomous regions ranked from rich to poor by GDP per capita are Shanghai, Beijing, Tianjin, Zhejiang, Guangdong, Jiangsu, Fujian, Liaoning, Shandong, Heilongjiang, Hebei, Xinjiang, Hubei, Jilin, Hainan, Inner Mongolia, Hunan, Qinghai, Henan, Chongqing, Shanxi, Ningxia,Tibet, Anhui, Jiangxi, Sichuan, Shaanxi, Guangxi, Yunnan, Gansu, Guizhou. The first 10 provinces are grouped as rich. The 21 provinces which follow are group as poor. Mainland China is divided into 6 regions as follows; Northern China includes 5 provinces: Beijing, Tianjin, Hebei, Shanxi, Inner Mongolia; Northeastern China includes 3 provinces: Liaoning, Jilin, Heilongjiang; Eastern China includes 7 provinces: Shanghai, Jiangsu, Zhejiang, Anhui, Fujian, Jiangxi, Shandong; Central and Southern China includes 6 provinces: Henan, Hubei, Hunan, Guangdong, Guangxi, Hainan; Southwestern China includes 5 provinces: Chongqing, Sichuan, Guizhou, Yunnan, Tibet; Northwestern China includes 5 provinces: Shaanxi, Gansu, Qinghai, Ningxia, Xinjiang. The Eastern Coastal zone includes 12 provinces: Beijing, Tianjin, Hebei, Liaoning, Shanghai, Jiangsu, Zhejiang, Fujian, Shandong, Guangdong, Guangxi, Hainan. The Central zone includes 9 provinces: Shanxi, Inner Mongolia, Jilin, Heilongjiang, Anhui, Jiangxi, Henan, Hubei, Hunan. The Western zone includes 10 provinces: Chongqing, Sichuan, Guizhou, Yunnan, Tibet, Shaanxi, Gansu, Qinghai, Ningxia, Xinjiang. The Western Development zone includes 12 provinces as Inner Mongolia, Guangxi, and western zone. In Tables 2 and 3 and in the discussion that follows, EC - CW zones denote Eastern Coastal zone - Central and Western zones, EC - WD zones denote Eastern and Central zone - Western Development zone, E - C - W zones denote Eastern zone Central zone - Western zone.
} 
The picture that emerges from Table 4 is that Hukou registration is a significant policy impediment preventing the achievement of a more equal distribution of incomes and wages in China. The number of people who would migrate across regions after its removal is between 200 and 600 million in the first 4 cases ( $\frac{1}{6}$ to $\frac{1}{2}$ of the population of China). Unlike the international migration case where Hamilton and Whalley (1984) found very large efficiency effects due to larger initial wage disperssion, the efficiency effects are more modest but still significant. The gain is $15 \%$ of national output in the first case, and around $1.7-3.2 \%$ in the other three cases.

Table 5 reports the more detailed 31 region model calculation. An increase in output of around $7 \%$ results, and differentials in both GDP / capita and income per worker across regions are sharply narrowed. Pairwise migration between provinces is smaller, and migration in aggregate falls. The direction of migration is from poor to rich provinces, that is, from Henan, Guizhou, Sichuan, Anhui, Guangxi, Yunnan, Shaanxi, Gansu, Hunan, Chongqing, Jiangxi, Hebei, Shanxi to Guangdong, Shanghai, Beijing, Zhejiang, Jiangsu, Fujian, Tianjin. Again the overall picture converged by these results is similar, removal of Hukou registration would play a major role in reducing relative income inequality in China. 
Table 2. Data Used to Calibrate 2, 3, and 6 Region Formulations of Chinese Inter-regional Labour Mobility Model (All Data for 2001 ${ }^{1}$ )

\section{$\underline{\text { Regional Classification }}{ }^{2}$ Used in Model Variants $^{-1}$}

\begin{tabular}{|c|c|c|c|c|c|c|}
\hline & Urban - Rural & Rich - Poor & $\mathrm{EC}-\mathrm{CW}$ & $\mathrm{EC}-\mathrm{WD}$ & $E-C-W$ & 6 Regions $^{3}$ \\
\hline \multirow{4}{*}{$\begin{array}{c}\text { GDP per capita } \\
\text { by Region }\end{array}$} & \multirow{3}{*}{ U 12.38835} & \multirow{3}{*}{ R 13.29862} & \multirow{3}{*}{ EC 12.08961} & \multirow{3}{*}{ EC 9.77881} & \multirow{3}{*}{ E 12.08961} & $\mathrm{NC} \quad 9.22246$ \\
\hline & & & & & & NEC 9.93508 \\
\hline & & & & & & EC 11.03664 \\
\hline & & & & & C 6.36075 & CSC 7.78470 \\
\hline \multirow[t]{2}{*}{$\left(10^{3} \mathrm{RMB}\right)$} & & & & & & SWC 4.71466 \\
\hline & R 6.05665 & P 5.79754 & CW $\quad 5.79994$ & WD 5.03428 & W 4.94210 & NSC 5.43888 \\
\hline \multirow{4}{*}{$\begin{array}{l}\text { Wage Rate } \\
\text { by Region }\end{array}$} & \multirow{3}{*}{ U 16.63805} & \multirow{3}{*}{ R 11.30238} & \multirow{3}{*}{ EC 10.40758} & \multirow{3}{*}{ EC 9.24304} & \multirow{2}{*}{ E 10.40758} & NC $\quad 9.61953$ \\
\hline & & & & & & NEC 8.55412 \\
\hline & & & & & & EC $\quad 9.82212$ \\
\hline & & & & & C 7.28020 & CSC 8.01942 \\
\hline \multirow[t]{2}{*}{$\left(10^{3} \mathrm{RMB}\right)$} & & & & & & SWC 6.04259 \\
\hline & R 5.73975 & P 6.71116 & CW $\quad 6.77753$ & WD 5.99797 & W 6.03203 & NSC 6.00533 \\
\hline \multirow{4}{*}{$\begin{array}{l}\text { Work Force } \\
\text { by Region }\end{array}$} & \multirow{3}{*}{ U 142.236} & \multirow{3}{*}{ R 218.465} & \multirow{3}{*}{ EC 264.782} & \multirow{3}{*}{ EC 447.666} & \multirow{3}{*}{ E 264.782} & $\mathrm{NC} \quad 68.458$ \\
\hline & & & & & & NEC 45.216 \\
\hline & & & & & & EC $\quad 187.020$ \\
\hline & & & & & C 218.451 & CSC 182.539 \\
\hline \multirow[t]{2}{*}{ (10 $10^{6}$ People) } & & & & & & SWC 105.539 \\
\hline & R 482.291 & P 412.062 & CW 365.745 & WD 182.861 & W 147.294 & NSC $\quad 41.755$ \\
\hline \multirow{4}{*}{$\begin{array}{l}\text { Population } \\
\text { by Region }\end{array}$} & \multirow{3}{*}{$\mathrm{U} 472.20$} & \multirow{3}{*}{ R 442.38} & \multirow{3}{*}{ EC 527.10} & \multirow{3}{*}{ EC 903.36} & \multirow{3}{*}{ E 527.10} & $\mathrm{NC} \quad 147.35$ \\
\hline & & & & & & NEC 106.96 \\
\hline & & & & & & EC $\quad 365.77$ \\
\hline & & & & & C 447.91 & CSC 354.93 \\
\hline \multirow[t]{2}{*}{ (10 $0^{6}$ People) } & & & & & & SWC 200.86 \\
\hline & R 795.63 & P 825.45 & CW 740.73 & WD 364.47 & W 292.82 & NSC 91.96 \\
\hline
\end{tabular}

1. All data are drawn from Chinese Statistical Yearbook (2002).

2. Regional classifications are given in Footnote 5 (Page 7).

3. NC, NEC, EC, CSC, SWC, and NSC denote Northern China (5 provinces), Northeastern China (3 provinces), Eastern China (7 provinces), Central and Southern China (6 provinces), Southwestern China (5 provinces), and Northwestern China (5 provinces), respectively. 
Table 3. Data Used to Calibrate 31 Province Version of Chinese Inter-regional Labour Mobility Model (All Data for 2001 ${ }^{1}$ )

\begin{tabular}{|c|c|c|c|c|}
\hline Province & $\begin{array}{c}\text { GDP per capita } \\
\text { by Region } \\
\left(10^{3} \mathrm{RMB}\right)\end{array}$ & $\begin{array}{l}\text { Wage Rate } \\
\text { by Region } \\
\left(10^{3} \mathrm{RMB}\right)\end{array}$ & $\begin{array}{c}\text { Work Force } \\
\text { by Region } \\
\left(10^{6} \text { People }\right)\end{array}$ & $\begin{array}{l}\text { Population } \\
\text { by Region } \\
\left(10^{6} \text { People }\right)\end{array}$ \\
\hline Beijing & 20575.92 & 21.64844 & 6.295 & 13.83 \\
\hline Tianjin & 18327.69 & 17.17241 & 4.105 & 10.04 \\
\hline Hebei & 8326.29 & 7.79436 & 33.796 & 66.99 \\
\hline Shanxi & 5440.01 & 7.65554 & 14.129 & 32.72 \\
\hline Inner Mongolia & 6503.11 & 7.91288 & 10.133 & 23.77 \\
\hline Liaoning & 12000.67 & 8.76288 & 18.334 & 41.94 \\
\hline Jilin & 7552.88 & 8.71343 & 10.572 & 26.91 \\
\hline Heilongjiang & 9344.00 & 8.21618 & 16.310 & 38.11 \\
\hline Shanghai & 30674.35 & 26.25800 & 6.924 & 16.14 \\
\hline Jiangsu & 12932.58 & 10.71770 & 35.654 & 73.55 \\
\hline Zhejiang & 14628.55 & 11.47211 & 27.720 & 46.13 \\
\hline Anhui & 5199.32 & 6.44760 & 33.897 & 63.28 \\
\hline Fujian & 12365.35 & 10.90519 & 16.778 & 34.40 \\
\hline Jiangxi & 5197.52 & 7.61850 & 19.331 & 41.86 \\
\hline Shandong & 10439.45 & 8.69493 & 46.716 & 90.41 \\
\hline Henan & 5714.40 & 5.79073 & 55.166 & 95.55 \\
\hline Hubei & 7802.98 & 9.08476 & 24.525 & 59.75 \\
\hline Hunan & 6038.51 & 7.78799 & 34.388 & 65.96 \\
\hline Guangdong & 13680.73 & 12.53207 & 39.629 & 77.83 \\
\hline Guangxi & 4868.82 & 5.03787 & 25.434 & 47.88 \\
\hline Hainan & 6858.79 & 8.54323 & 3.397 & 7.96 \\
\hline Chongqing & 5649.89 & 7.31835 & 16.240 & 30.97 \\
\hline Sichuan & 5117.78 & 7.25216 & 44.146 & 86.40 \\
\hline Guizhou & 2855.75 & 3.89057 & 20.682 & 37.99 \\
\hline Yunnan & 4839.54 & 4.64526 & 23.225 & 42.87 \\
\hline Tibet & 5274.90 & 8.32565 & 1.246 & 2.63 \\
\hline Shaanxi & 5040.37 & 5.00624 & 17.846 & 36.59 \\
\hline Gansu & 4165.09 & 4.99672 & 11.872 & 25.75 \\
\hline Qinghai & 5754.30 & 7.06371 & 2.403 & 5.23 \\
\hline Ningxia & 5299.82 & 6.65314 & 2.780 & 5.63 \\
\hline Xinjiang & 7918.34 & 9.71990 & 6.854 & 18.76 \\
\hline
\end{tabular}


Table 4. Impacts of Hukou Elimination in Alternative Regional Divides in Inter-regional Labour Mobility Model for China (Data for 2001 ${ }^{1}$ )

\begin{tabular}{|c|c|c|c|c|c|c|}
\hline & Urban - Rural & Rich - Poor & $\mathrm{EC}-\mathrm{CW}$ & EC - WD & $E-C-W$ & 6 Regions \\
\hline \multirow{17}{*}{$\begin{array}{c}\text { Output } \\
\left(10^{6} \mathrm{RMB}\right)\end{array}$} & \multicolumn{6}{|l|}{ Base Case } \\
\hline & U 5849.777 & R 5883.043 & EC 6372.436 & EC 8833.782 & E 6372.436 & $\begin{array}{ll}\mathrm{NC} & 1358.929\end{array}$ \\
\hline & R 4818.849 & P 4785.583 & CW 4296.190 & WD 1834.844 & C 2849.044 & NEC 1062.656 \\
\hline & & & & & W 1447.146 & EC $\quad 4036.870$ \\
\hline & & & & & & CSC 2763.025 \\
\hline & & & & & & SWC 946.987 \\
\hline & & & & & & NSC 500.159 \\
\hline & $Y=10668.626$ & $Y=10668.626$ & $Y=10668.626$ & $Y=10668.626$ & $Y=10668.626$ & $Y=10668.626$ \\
\hline & \multicolumn{6}{|c|}{ After Hukou Elimination } \\
\hline & U 9531.818 & R 7395.960 & EC 7615.861 & EC 9718.435 & E 7614.698 & NC $\quad 1567.992$ \\
\hline & R 2730.995 & P 3615.498 & CW 3292.926 & WD 1133.458 & C 2436.998 & NEC 1084.038 \\
\hline & & & & & W 881.467 & EC $\quad 4664.376$ \\
\hline & & & & & & CSC 2671.985 \\
\hline & & & & & & SWC 496.865 \\
\hline & & & & & & NSC 362.945 \\
\hline & $Y=12082.813$ & $Y=11011.457$ & $Y=10908.787$ & $Y=10851.893$ & $Y=10993.163$ & $Y=10848.200$ \\
\hline & $\Delta=1414.187$ & $\Delta=342.831$ & $\Delta=240.161$ & $\Delta=183.267$ & $\Delta=324.537$ & $\Delta=179.574$ \\
\hline \multirow{17}{*}{$\begin{array}{c}\text { Average } \\
\text { Income } \\
\text { (GDP } \\
\text { per capita) } \\
\left(10^{3} \mathrm{RMB}\right)\end{array}$} & \multicolumn{6}{|l|}{ Base Case } \\
\hline & U 12.38835 & R 13.29862 & EC 12.08961 & EC 9.77881 & E 12.08961 & NC 9.22246 \\
\hline & $\mathrm{R} 6.05665$ & P 5.79754 & CW 5.79994 & WD 5.03428 & C 6.36075 & NEC 9.93508 \\
\hline & & & & & W 4.94210 & EC 11.03664 \\
\hline & & & & & & CSC 7.78470 \\
\hline & & & & & & SWC 4.71466 \\
\hline & & & & & & NSC 5.43888 \\
\hline & $\bar{I}=8.41487$ & $\bar{I}=8.41487$ & $\bar{I}=8.41487$ & $\bar{I}=8.41487$ & $\bar{I}=8.41487$ & $\bar{I}=8.41487$ \\
\hline & \multicolumn{6}{|c|}{ After Hukou Elimination } \\
\hline & U 8.89620 & R 9.71770 & EC 9.53256 & EC 8.79230 & E 9.54737 & NC $\quad 7.94761$ \\
\hline & R 12.60758 & P 7.13467 & CW 7.02263 & WD 6.97531 & C 7.18102 & NEC 9.62809 \\
\hline & & & & & W 6.73383 & EC 9.31484 \\
\hline & & & & & & CSC 8.04717 \\
\hline & & & & & & SWC 6.46802 \\
\hline & & & & & & NSC 7.50786 \\
\hline & $\bar{I}=9.53031$ & $\bar{I}=8.68528$ & $\bar{I}=8.60430$ & $\bar{I}=8.55942$ & $\bar{I}=8.62352$ & $\bar{I}=8.55651$ \\
\hline & $\Delta=1.11544$ & $\Delta=0.27041$ & $\Delta=0.18943$ & $\Delta=0.14455$ & $\Delta=0.20865$ & $\Delta=0.14164$ \\
\hline
\end{tabular}




\begin{tabular}{|c|c|c|c|c|c|c|}
\hline \multirow{17}{*}{$\begin{array}{c}\text { Income } \\
\text { per Worker } \\
\left(10^{3} \mathrm{RMB}\right)\end{array}$} & \multicolumn{6}{|l|}{$\underline{\text { Base Case }}$} \\
\hline & U 39.46259 & R 26.92900 & EC 24.06673 & EC 19.73298 & E 24.06673 & NC $\quad 19.85055$ \\
\hline & \multirow[t]{5}{*}{ R 9.99158} & \multirow[t]{5}{*}{ P 11.61375} & \multirow[t]{5}{*}{ CW 11.74641} & \multirow[t]{5}{*}{ WD 10.03409} & C 13.04203 & NEC 23.50177 \\
\hline & & & & & W 9.82488 & EC $\quad 21.58523$ \\
\hline & & & & & & CSC 15.13663 \\
\hline & & & & & & SWC 8.97286 \\
\hline & & & & & & NSC 11.97842 \\
\hline & $I=16.92017$ & $I=16.92017$ & $I=16.92017$ & $I=16.92017$ & $I=16.92017$ & $I=16.92017$ \\
\hline & \multicolumn{6}{|c|}{ After Hukou Elimination } \\
\hline & U 20.73320 & R 19.62456 & EC 19.04657 & EC 17.70725 & E 19.05042 & NC $\quad 17.04807$ \\
\hline & R 15.21683 & P 14.25360 & CW 14.27526 & WD 13.87542 & C 14.75840 & NEC 22.69771 \\
\hline & & & & & W 13.41819 & EC 18.15549 \\
\hline & & & & & & CSC 15.59347 \\
\hline & & & & & & SWC 12.26774 \\
\hline & & & & & & NSC 16.47855 \\
\hline & $I=19.16304$ & $I=17.46389$ & $I=17.30106$ & $I=17.21083$ & $I=17.33972$ & $I=17.20497$ \\
\hline & $\Delta=2.24287$ & $\Delta=0.54372$ & $\Delta=0.38089$ & $\Delta=0.29066$ & $\Delta=0.41955$ & $\Delta=0.28480$ \\
\hline \multirow{9}{*}{$\begin{array}{l}\text { Wage Rate } \\
\left(10^{3} \mathrm{RMB}\right)\end{array}$} & \multicolumn{6}{|l|}{$\underline{\text { Base Case }}$} \\
\hline & U 16.63805 & R 11.30238 & EC 10.40758 & EC 9.24304 & E 10.40758 & NC $\quad 9.61953$ \\
\hline & R 5.73975 & P 6.71116 & CW $\quad 6.77753$ & WD 5.99797 & C 7.28020 & NEC 8.55412 \\
\hline & & & & & W 6.03203 & EC $\quad 9.82212$ \\
\hline & & & & & & CSC 8.01942 \\
\hline & & & & & & SWC 6.04259 \\
\hline & & & & & & NSC 6.00533 \\
\hline & \multicolumn{6}{|c|}{ After Hukou Elimination } \\
\hline & $W=8.74144$ & $W=8.74144$ & $W=8.74144$ & $W=8.74144$ & $W=8.74144$ & $W=8.74144$ \\
\hline Change in & U 302.81913 & R 158.40700 & EC 135.07144 & EC 101.17314 & E 134.93051 & NC 23.51675 \\
\hline Work Force & $\mathrm{R}-302.81913$ & P - 158.40700 & CW -135.07144 & WD -101.17314 & C -53.32496 & NEC 2.54381 \\
\hline by Region & & & & & W -81.60556 & EC $\quad 69.89272$ \\
\hline (Migration) & & & & & & CSC -11.18622 \\
\hline (millions of & & & & & & SWC-65.03726 \\
\hline people) & & & & & & NSC - 19.72979 \\
\hline Change in & U 579.01525 & R 318.69952 & EC 271.82831 & EC 201.97491 & E 270.46951 & NC $\quad 49.94098$ \\
\hline Population & $\mathrm{R}-579.01525$ & P -318.69952 & CW -271.82831 & WD -101.97491 & C - 108.54383 & NEC $\quad 5.63122$ \\
\hline by Region & & & & & W -161.92568 & EC 134.97694 \\
\hline (Migration) & & & & & & CSC -22.88965 \\
\hline (millions of & & & & & & SWC-124.04133 \\
\hline people) & & & & & & NSC -43.61816 \\
\hline
\end{tabular}


Table 5. The Impacts of Hukou Elimination in a Simple Model of Inter-regional Labour Mobility Applied to All 31 Regions of China (Data for 2001 ${ }^{1}$ )

\begin{tabular}{|c|c|c|c|c|c|c|}
\hline Province & $\begin{array}{c}\text { Base Case } \\
\text { Output } \\
\left(10^{6} \mathrm{RMB}\right)\end{array}$ & $\begin{array}{c}\text { Output } \\
\text { after Hukou } \\
\text { Elimination } \\
\left(10^{6} \mathrm{RMB}\right)\end{array}$ & $\begin{array}{l}\text { Change in } \\
\text { Output } \\
\left(10^{6} \mathrm{RMB}\right)\end{array}$ & $\begin{array}{c}\text { Base Case } \\
\text { GDP per capita } \\
\left(10^{3} \mathrm{RMB}\right)\end{array}$ & $\begin{array}{c}\text { GDP per capita } \\
\text { after Hukou } \\
\text { Elimination } \\
\left(10^{3} \mathrm{RMB}\right)\end{array}$ & $\begin{array}{c}\text { Change in } \\
\text { GDP per capita } \\
\left(10^{3} \mathrm{RMB}\right)\end{array}$ \\
\hline Beijing & 284.565 & 674.33385 & 389.76885 & 20.57592 & 8.26510 & - 12.31082 \\
\hline Tianjin & 184.010 & 285.46995 & 101.45995 & 18.32769 & 9.28094 & - 9.04675 \\
\hline Hebei & 557.778 & 517.97596 & -37.80204 & 8.32629 & 9.28937 & 0.96308 \\
\hline Shanxi & 177.997 & 152.29076 & -25.70624 & 5.44001 & 6.17929 & 0.73928 \\
\hline Inner Mongolia & 154.579 & 143.71191 & - 10.86709 & 6.50311 & 7.14664 & 0.64353 \\
\hline Liaoning & 503.308 & 511.49091 & 8.18291 & 12.00067 & 11.90896 & - 0.09171 \\
\hline Jilin & 203.248 & 208.14776 & 4.89976 & 7.55288 & 7.53770 & 0.01518 \\
\hline Heilongjiang & 356.100 & 349.70735 & -6.39265 & 9.34400 & 9.88959 & 0.54559 \\
\hline Shanghai & 495.084 & 954.89118 & 459.80718 & 30.67435 & 10.15850 & -20.51585 \\
\hline Jiangsu & 951.191 & 1114.35970 & 163.16870 & 12.93258 & 10.49297 & - 2.43961 \\
\hline Zhejiang & 674.815 & 884.65167 & 209.83667 & 14.62855 & 11.08851 & -3.54004 \\
\hline Anhui & 329.013 & 191.92121 & - 137.09179 & 5.19932 & 7.01235 & 1.81303 \\
\hline Fujian & 425.368 & 514.91721 & 89.54921 & 12.36535 & 9.86026 & -2.50509 \\
\hline Jiangxi & 217.568 & 174.40481 & - 43.16139 & 5.19752 & 5.93255 & 0.73503 \\
\hline Shandong & 943.831 & 962.99965 & 19.16865 & 10.43945 & 10.44138 & 0.00118 \\
\hline Henan & 546.011 & 319.58354 & - 226.42746 & 5.71440 & 8.58129 & 2.86689 \\
\hline Hubei & 466.228 & 497.29110 & 31.06310 & 7.80298 & 7.46900 & - 0.33398 \\
\hline Hunan & 398.300 & 335.53822 & -62.76178 & 6.03851 & 6.74248 & 0.70397 \\
\hline Guangdong & 1064.771 & 1500.15079 & 435.37979 & 13.68073 & 9.49296 & -4.18777 \\
\hline Guangxi & 233.119 & 123.71032 & - 109.40868 & 4.86882 & 8.40411 & 3.53529 \\
\hline Hainan & 54.596 & 55.15710 & 0.56110 & 6.85879 & 6.98138 & 0.12259 \\
\hline Chongqing & 174.977 & 128.51436 & -46.46264 & 5.64989 & 6.71339 & 1.06350 \\
\hline Sichuan & 442.176 & 294.55856 & - 147.61744 & 5.11778 & 6.13662 & 1.01884 \\
\hline Guizhou & 108.490 & 11.63620 & -96.85380 & 2.85575 & 6.38296 & 3.52721 \\
\hline Yunnan & 207.471 & 108.27470 & - 99.19630 & 4.83954 & 9.05960 & 4.22006 \\
\hline Tibet & 13.873 & 13.19897 & -0.67403 & 5.27490 & 5.50943 & 0.23453 \\
\hline Shaanxi & 184.427 & 112.56756 & - 71.85944 & 5.04037 & 8.75520 & 3.71483 \\
\hline Gansu & 107.251 & 55.84026 & -51.41074 & 4.16509 & 7.24860 & 3.08351 \\
\hline Qinghai & 30.095 & 23.80752 & -6.28748 & 5.75430 & 7.08394 & 1.32964 \\
\hline Ningxia & 29.838 & 20.14029 & -9.69771 & 5.29982 & 6.92708 & 1.62726 \\
\hline Xinjiang & 148.548 & 166.19509 & 17.64709 & 7.91834 & 7.08414 & - 0.83420 \\
\hline Nation & 10668.626 & 11407.43845 & 738.81245 & 8.41487 & 8.99761 & 0.58274 \\
\hline
\end{tabular}




\begin{tabular}{|c|c|c|c|c|c|c|}
\hline $\begin{array}{l}\text { Base Case } \\
\text { Income } \\
\text { per Worker } \\
\left(10^{3} \mathrm{RMB}\right)\end{array}$ & $\begin{array}{c}\text { Income } \\
\text { per Worker } \\
\text { after Hukou } \\
\text { Elimination } \\
\left(10^{3} \mathrm{RMB}\right)\end{array}$ & $\begin{array}{l}\text { Change in } \\
\text { Income } \\
\text { per Worker } \\
\left(10^{3} \mathrm{RMB}\right)\end{array}$ & $\begin{array}{l}\text { Base Case } \\
\text { Wage Rate }\end{array}$ & $\begin{array}{l}\text { Wage Rate } \\
\text { after Hukou } \\
\text { Elimination } \\
\left(10^{3} \mathrm{RMB}\right)\end{array}$ & $\begin{array}{l}\text { Work Force } \\
\text { Migration }\end{array}$ & $\begin{array}{l}\text { Population } \\
\text { Migration }\end{array}$ \\
\hline 45.20492 & 17.67941 & -27.52551 & 21.64844 & 8.46659 & 31.84733 & 67.75814 \\
\hline 44.82582 & 22.10069 & -22.72513 & 17.17241 & 8.46659 & 8.81179 & 20.71874 \\
\hline 16.50426 & 17.92770 & 1.42344 & 7.79436 & 8.46659 & -4.90359 & - 11.22995 \\
\hline 12.59799 & 13.93265 & 1.33466 & 7.65554 & 8.46659 & -3.19851 & -8.07465 \\
\hline 15.25501 & 16.32250 & 1.06749 & 7.91288 & 8.46659 & -1.32847 & -3.66100 \\
\hline 27.45217 & 26.52396 & -0.92821 & 8.76288 & 8.46659 & 0.95011 & 1.01009 \\
\hline 19.22512 & 18.68050 & -0.54462 & 8.71343 & 8.46659 & 0.57051 & 0.70424 \\
\hline 21.83323 & 22.49866 & 0.66543 & 8.21618 & 8.46659 & -0.76653 & -2.74884 \\
\hline 71.50260 & 23.05523 & - 48.44737 & 26.25796 & 8.46659 & 34.49355 & 77.85922 \\
\hline 26.67838 & 21.07494 & -5.60344 & 10.71770 & 8.46659 & 17.22205 & 32.65057 \\
\hline 24.34398 & 17.96622 & -6.37776 & 11.47211 & 8.46659 & 21.51972 & 33.65095 \\
\hline 9.70626 & 12.74566 & 3.03930 & 6.44760 & 8.46659 & - 18.83923 & - 35.91098 \\
\hline 25.35272 & 19.68339 & -5.66933 & 10.90519 & 8.46659 & 9.38198 & 17.82147 \\
\hline 11.25488 & 12.50776 & 1.25288 & 7.61850 & 8.46659 & -5.38728 & -12.46205 \\
\hline 20.20359 & 19.67302 & -0.53057 & 8.69493 & 8.46659 & 2.23427 & 1.82576 \\
\hline 9.89760 & 14.47122 & 4.57362 & 5.79073 & 8.46659 & - 33.08192 & -58.30810 \\
\hline 19.01032 & 17.71677 & - 1.29355 & 9.08476 & 8.46659 & 3.54395 & 6.83072 \\
\hline 11.58253 & 12.59176 & 1.00923 & 7.78799 & 8.46659 & - 7.74056 & - 16.19516 \\
\hline 26.86848 & 18.15219 & - 8.71629 & 12.53207 & 8.46659 & 43.01395 & 80.19768 \\
\hline 9.16564 & 15.40369 & 6.23805 & 5.03787 & 8.46659 & - 17.40278 & - 33.15979 \\
\hline 16.07183 & 15.92766 & -0.14417 & 8.54323 & 8.46659 & 0.06597 & 0.05940 \\
\hline 10.77445 & 12.46494 & 1.69049 & 7.31835 & 8.46659 & -5.92994 & -11.82700 \\
\hline 10.01622 & 11.69352 & 1.68730 & 7.25216 & 8.46659 & - 18.95610 & - 38.39989 \\
\hline 5.24562 & 11.41543 & 6.16981 & 3.89057 & 8.46659 & - 19.66266 & - 36.16699 \\
\hline 8.93309 & 16.28171 & 7.34862 & 4.64526 & 8.46659 & - 16.57492 & - 30.91862 \\
\hline 11.13403 & 11.32238 & 0.18835 & 8.32565 & 8.46659 & -0.08030 & -0.23439 \\
\hline 10.33436 & 17.47756 & 7.14320 & 5.00624 & 8.46659 & - 11.40531 & - 23.73277 \\
\hline 9.03395 & 15.30737 & 6.27342 & 4.99672 & 8.46659 & -8.22407 & -18.04640 \\
\hline 12.52393 & 15.01123 & 2.58730 & 7.06371 & 8.46659 & -0.81702 & - 1.86923 \\
\hline 10.73309 & 13.65863 & 2.92554 & 6.65314 & 8.46659 & -1.30545 & -2.72253 \\
\hline 21.67318 & 18.87859 & - 2.79459 & 9.71990 & 8.46659 & 1.94936 & 4.70015 \\
\hline 16.92017 & 18.09191 & 1.17174 & & & & \\
\hline
\end{tabular}




\section{EXTENDING THE BASIC MODEL TO CAPTURE EFFICIENCY DIFFERENCES ACROSS INDIVIDUALS AND INEQUALITY WITHIN REGIONS}

The model set out above can be elaborated on to also incorporate within region efficiency differences across individuals and hence inequality within regions both before and after the elimination of Hukou. With these features present, wage differentials across individuals will not disappear with the elimination of Hukou registration as in the basic model presented above.

To incorporate efficiency differences across individuals within regions into the model we use a functional form for the distribution of income within regions which through calibration implies a distribution of the efficiencies of labour across individuals within a region. This approach allows us to specify initial differences in efficiencies across all individuals in specific regions of the economy so as to also calibrate a modified version of the simple model to within region inequality as measured by Gini coefficients for each region, as well as to a national Gini inequality measure of income inequality. In this model variant removing labour mobility restrictions once again equalizes wage rates per efficiency unit of labour across regions, but with differing endowments of efficiency units of labour across individuals wage rate inequality will remain both across individuals and across regions when Hukou restrictions are removed. An elimination of Hukou restrictions will be equalizing, but compete equality of wage rates across individuals within regions will not be achieved as efficiency differences remain.

The model we use for this case can be formally stated as follows. The same equations (3) - (7) characterize the production side of the model, but there are now also distributions of income within regions which reflect differences in the efficiency of labour within regions and these need to be incorporated. Denoting $N_{s}$ as the number of people in region $s$, and $L_{s}$ as labour in efficiency units in region $s\left(N_{s} \neq L_{s}\right)$, we have average region incomes $\bar{I}_{s}$, national income $I$, average national income $\bar{I}$, and the average region wage $\bar{W}_{s}$ as in (8) - (10) above.

The functional form for the income distribution within each region $s$ can take many forms. For simplicity, we assume a non-linear form for the regional income distribution as

$$
I_{s}^{n}=C_{s}+D_{s} n+E_{s} n^{\delta_{s}}, \quad n=1, \cdots, N_{s}, \quad s=1, \cdots, S .
$$

where $n$ is an index $1, \cdots, N_{s}$ across the individuals $N_{s}$ in regions ranked from poor to rich, and $C_{s}, D_{s}$, $E_{s}$ and $\delta_{s}$ are parameters of the distribution function. This provides sufficient free parameters to calibrate the model to both regional and national Gini coefficients through a generated distribution of efficiencies. If a simple linear form were used there would not insufficient free parameters for calibration.

Using realtion (11), we calibrate the model to satisfy $2 S+1$ conditions reflecting total income and Gini coefficient constraints and in addition use the same calibration conditions for the simple model above. Since

$$
\begin{gathered}
I_{s}=\sum_{n=1}^{N_{s}} I_{s}^{n}=\sum_{n=1}^{N_{s}}\left[C_{s}+D_{s} n+E_{s} n^{\delta_{s}}\right], \quad s=1, \cdots, S, \\
g_{s}=\frac{1}{2 N_{s}^{2} \bar{I}_{s}} \sum_{n=1}^{N_{s}} \sum_{n^{\prime}=1}^{N_{s}}\left|I_{s}^{n}-I_{s}^{n^{\prime}}\right|=1-\frac{1}{N_{s}^{2} \bar{I}_{s}} \sum_{n=1}^{N_{s}} \sum_{n^{\prime}=1}^{N_{s}} \min \left\{I_{s}^{n}, I_{s}^{n^{\prime}}\right\}, \quad s=1, \cdots, S
\end{gathered}
$$

where $g_{s}$ is the region $s$ Gini coefficient in incomes ${ }^{6}$.

\footnotetext{
${ }^{6}$ See Sen $(1972)$
} 
The national Gini coefficient, $g$, is given by

$$
\begin{aligned}
g & =\frac{1}{2 N^{2} \bar{I}}\left\{\sum_{s=1}^{S} \sum_{n=1}^{N_{s}} \sum_{n^{\prime}=1}^{N_{s}}\left|I_{s}^{n}-I_{s}^{n^{\prime}}\right|+2 \sum_{s \neq s^{\prime}} \sum_{n=1}^{N_{s}} \sum_{n^{\prime}=1}^{N_{s^{\prime}}}\left|I_{s}^{n}-I_{s^{\prime}}^{n^{\prime}}\right|\right\} \\
& =1-\frac{1}{N^{2} \bar{I}}\left\{\sum_{s=1}^{S} \sum_{n=1}^{N_{s}} \sum_{n^{\prime}=1}^{N_{s}} \min \left\{I_{s}^{n}, I_{s}^{n^{\prime}}\right\}+2 \sum_{s \neq s^{\prime}} \sum_{n=1}^{N_{s}} \sum_{n^{\prime}=1}^{N_{s^{\prime}}} \min \left\{I_{s}^{n}, I_{s^{\prime}}^{n^{\prime}}\right\}\right\}
\end{aligned}
$$

One difficulty in executing these procedures is that initial data on regional and national Gini coefficients and estimates of regional and national GDP / capita can be matually inconsistent. Where this occurs, we have adopted a procedure of accepting data on region and a national GDP / capita, and accepting one or more of the Gini coefficient estimates from Chang (2002) for 2000 and setting remaining Gini coefficients at bounds implied by mutual consistency requirements. In this main, there are close to the available literature estimates, but do depart from these slightly. No other procedure short of modifying other portions of input data seems to suggest itself.

For the urban - rural case above where $S=2, I_{U}=5849.777, I_{R}=4818.847$ and $I=10668.626$ from Section 2. If the three Gini coefficients (drawing on Table 1 in Section 2, Chang (2002) and Liu, Yao and Zhang (2001)) are set as $g_{U}=0.3200, g_{R}=0.3500$ and $g=0.4600$, use all these data can be used as inputs in to calibration. This allows us to calibrate the model for $8(=4 \times 2)$ unknown distribution function parameters: $C_{s}, D_{s}, E_{s}$, and $\delta_{s}$ for $s=U, R$. If we then remove Hukou restrictions and compute a new model solution the three computed Gini coefficients are $g_{U}=0.274062, g_{R}=0.282343$ and $g=0.293886$. Compared to the case of the simple model, the impact on inequality of Hukou removal is smaller, but it is still significant and especially so for national inequality.

For the rich - poor case above $S$ also equals 2 , and $I_{R}=5883.043$ and $I_{P}=4785.583, I=10668.626$ (from Section 2). Drawing again on Table 1 in Section 2, Chang (2002) and Liu, Yao and Zhang (2001) we now set the three Gini coefficients to be $g_{R}=0.4094, g_{P}=0.2030$, and $g=0.4600$. We again calibrate the model and compute the $8(=4 \times 2)$ unknown parameters: $C_{s}, D_{s}, E_{s}$, and $\delta_{s}$ for $s=R, P$, and remove Hukou restrictions computing a new model solution. The three Gini coefficients in this solution are $g_{R}=0.246542$, $g_{P}=0.179664$, and $g=0.261539$. Impacts on inequality in this case are once again smaller compared to the simple model but still significant. Impacts on the three Gini coefficients differ from the urban - rural case because the initial dispersion in Gini coefficients is larger. For the eastern coastal - central and western case again $S=2, I_{E C}=6372.436, I_{C W}=4296.190$ and $I=10668.626$ from Section 2. Appealing again Table 1 in Section 2, Chang (2002) and Liu, Yao and Zhang (2001) we now set the three Gini coefficients as $g_{E C}=0.4119, g_{C W}=0.2040$ and $g=0.4600$. Using these, we again calibrate the model and remove Hukou restrictions. The three Gini coefficients are $g_{E C}=0.237945, g_{C W}=0.181590$, and $g=0.248018$. For the eastern central - western development 2 region case, $I_{E C}=8833.782, I_{W D}=1844.844$ and $I=10668.626$. The three Gini coefficients are set as $g_{E C}=0.4186, g_{W D}=0.1600$ and $g=0.4600$. Removing Hukou restrictions gives the three Gini coefficients $g_{E C}=0.242071, g_{W D}=0.148267$, and $g=0.280218$.

For the 3 region eastern - central - western case $S=3$, and $I_{E}=6372.436, I_{C}=2849.044, I_{W}=1447.146$ and $I=10668.626$ (from Section 2). From Table 1 in Section 2, Chang (2002) and Liu, Yao and Zhang (2001), we now set the three Gini coefficients as $g_{E}=0.4226, g_{C}=0.1440, g_{W}=0.1600$ and $g=0.4600$. Using these data, we calibrate the model to $12(=4 \times 3)$ unknown distribution parameters: $C_{s}, D_{s}, E_{s}$, and 
$\delta_{s}$ for $s=E, C, W$. If we remove Hukou restrictions and compute a new model solution in this case the three Gini coefficients are $g_{E}=0.174241, g_{E}=0.147957, g_{W}=0.156591$, and $g=0.200101$.

We have consolidated these results into a single Table (Table 6) which reports base case and counterfactual Gini coefficients for this extended model now applied to four alternative regional divides (urban - rural, rich - poor, and eastern coastal - central and western provinces). Impacts of Hukou removal on inequality as represented by the Gini coefficients are significant, but impacts are reduced relative to the simple model set out above since the dispersion in efficiencies across individuals implies that some inequality in wage rates remains after Hukou removal. Qualitatively this model behaves much as the simple model above.

Table 6. Effects of Hukou Elimination on Regional and National Gini Coefficients Using a Model with Distributions of Efficiencies within Regions

Regional Divide in Model Variant and Data

\begin{tabular}{|c|c|c|c|c|}
\hline Urban - Rural & Rich - Poor & $\mathrm{EC}-\mathrm{CW}$ & EC - WD & $E-C-W$ \\
\hline \multicolumn{5}{|c|}{ Gini Coefficients before Hukou Removal } \\
\hline$G_{U}=0.3200$ & $G_{R}=0.4094$ & $G_{E C}=0.4119$ & $G_{E C}=0.4186$ & $G_{E}=0.4226$ \\
\hline$G_{R}=0.3500$ & $G_{P}=0.2030$ & $G_{C W}=0.2040$ & $G_{W D}=0.1600$ & $G_{C}=0.1440$ \\
\hline & & & & $G_{W}=0.1600$ \\
\hline$G=0.4600$ & $G=0.4600$ & $G=0.4600$ & $G=0.4600$ & $G=0.4600$ \\
\hline \multicolumn{5}{|c|}{ Gini Coefficients after Hukou Removal } \\
\hline$G_{U}=0.274062$ & $G_{R}=0.246542$ & $G_{E C}=0.237945$ & $G_{E C}=0.242071$ & $G_{E}=0.174241$ \\
\hline$G_{R}=0.282343$ & $G_{P}=0.179664$ & $G_{C W}=0.181590$ & $G_{W D}=0.148267$ & $G_{C}=0.147957$ \\
\hline & & & & $G_{W}=0.156591$ \\
\hline$G=0.293886$ & $G=0.261539$ & $G=0.248018$ & $G=0.280218$ & $G=0.200101$ \\
\hline
\end{tabular}




\section{EXTENDING THE MODEL TO CAPTURE HOUSE PRICE EFFECTS ON MIGRATION ACROSS REGIONS}

A key feature of the urban - rural divide in modern day China missing in the model variants discussed thus far is large differences in housing (apartment) prices between the larger cities and village communities. Apartments in Beijing routinely sell for US $\$ 200,000$, and with average annual household incomes of around US $\$ 2,000$ in the Beijing area, even at $5 \%$ the imputed income on apartments owned outright is typically larger than annual cash income. Thus, households who received apartments at low (or zero) prices in urban areas in the late 1980's or the early 1990's have a large advantage over rural dwellers who might wish to move to the cities following a removal of Hukou restrictions. In this case the effect of added inward migration will be to drive up urban house prices further and this house price effect will act to dampen additional migration. Less migration following Hukou elimination appears in models incorporating house price effects in contrast to models which do not incorporate them; but working with them involves the use of a more complex analytical structure.

We have modified the basic model set out in Section 2 to also incorporate house price effects stemming from additional urban rural migration. We capture impacts on urban house prices and rents on urban rural mobility decisions by using a general equilibrium model with goods and housing separatly identified, and with housing prices in urban and rural areas differing reflecting market segmentation due to location. In this case, removing Hukou restrictions generates labour flows from rural to urban areas, but when these are included increases in urban house prices retard additional migration. Labour flows under Hukou removal are smaller and significant further redistribution occurs between urban dwellers whose house prices rise and rural dwellers whose prices fall. Efficiency gains from Hukou removal will tend to be smaller in models with house price effects since the number of migrants will be smaller.

We present this model variant more formally as follows. We once again denote $L_{s}$ as the labour in region $s$ before Hukou removal. The regional production functions are again

$$
Y_{s}=A_{s} L_{s}^{\alpha_{s}}, \quad s=U, R
$$

and the regional wage rates, $W_{s}$, are given as

$$
W_{s}=P_{G} \frac{\partial Y_{s}}{\partial L_{s}}=P_{G} \alpha_{s} A_{s} L_{s}^{\alpha_{s}-1}, \quad s=U, R
$$

where $P_{G}$ is the goods price, and regional rents, $R_{s}$, are

$$
R_{s}=P_{G} Y_{s}-W_{s} L_{s}, \quad s=U, R .
$$

Income in region $s$ is $I_{s}$

$$
I_{s}=P_{G} Y_{s}, \quad s=U, R .
$$

National income is $I=\sum_{s} I_{s}$. Full employment of labour in this model again implies that

$$
\sum_{s} L_{s}=L
$$

where $L$ is the national endowment of labour. 
We assume that there is continuum of individuals uniformly distributed over a unidimensional interval $\left[t_{R}, t_{U}\right]$ for a parameter $t$ who vary in terms of their preferences towards goods and housing, and for whom $t$ is a preference function parameter varying across all individuals. $\hat{t}$ is the critical value of preference parameter such that urban individuals all have $t$ vaules above $\hat{t}$ and lie on the interval $\left[\hat{t}, t_{U}\right]$ and rural individuals all have $t$ vaules below $\hat{t}$ and lie on the interval index $\left[t_{R}, \hat{t}\right]$. The continuum of individuals $\left[t_{R}, t_{U}\right]$ are assumed to differ in preference shares parameters for Cobb - Douglas preference defined as

$$
V_{t}(G, H)=G^{1-t} H^{t}, \quad t \in\left[t_{R}, t_{U}\right]
$$

We assume for simplicity that all individuals in urban and rural areas have endowments that are similar insructive, but differ in size. Their incomes are

$$
\begin{array}{lll}
I_{t}^{U}=R_{U} X_{t}^{U}+W_{U} L_{U} X_{t}^{U}+P_{U} E_{U} X_{t}^{U}=\left(P_{G} Y_{U}+P_{U} E_{U}\right) X_{t}^{U}, & t \in\left[\hat{t}, t_{U}\right] \\
I_{t}^{R}=R_{R} X_{t}^{R}+W_{R} L_{U} X_{t}^{R}+P_{R} E_{R} X_{t}^{R}=\left(P_{G} Y_{R}+P_{R} E_{R}\right) X_{t}^{R}, & t \in\left[t_{R}, \hat{t}\right]
\end{array}
$$

where $X^{U}$ and $X^{R}$ are uniform distribution random variable on $\left[\hat{t}, t_{U}\right]$ and $\left[t_{R}, \hat{t}\right]$.

Utility maximization subject to a budget constraint in each case implies

$$
\begin{aligned}
& G_{t}^{U}=\frac{1-t}{P_{G}} I_{t}^{U} \quad \text { and } \quad H_{t}^{U}=\frac{t}{P_{U}} I_{t}^{U}, \quad t \in\left[\hat{t}, t_{U}\right] \\
& G_{t}^{R}=\frac{1-t}{P_{G}} I_{t}^{R} \quad \text { and } \quad H_{t}^{R}=\frac{t}{P_{R}} I_{t}^{R}, \quad t \in\left[t_{R}, \hat{t}\right]
\end{aligned}
$$

A general equilibrium in this model involves an equilibrium value $\hat{t}$, and the division of the population into urban and rural components is endogenously determined. Before Hukou removal an equilibrium is characterized by wage rates $W_{s}$, a goods price $P_{G}$, urban and rural house prices $P_{U}$ and $P_{R}$ such that the following conditions hold.

[1] (Good Market Clearing) $\quad Y_{U}+Y_{R}=\int_{\hat{t}}^{t_{U}} G_{t}^{U} d t+\int_{t_{R}}^{\hat{t}} G_{t}^{R} d t$;

[2] (House Market Clearing) $\quad \int_{\hat{t}}^{t_{U}} H_{t}^{U} d t=E_{U}$ and $\int_{t_{R}}^{\hat{t}} H_{t}^{R} d t=E_{R}$;

[3] (Labour Market Clearing) $\quad L_{U}+L_{R}=L$;

If we consider the case of Hukou removal, under the assumption that all individuals have identical amounts labour in urban and rural areas, then labour is allocated by region such that

$$
L_{U}=\frac{t_{U}-\hat{t}}{t_{U}-t_{R}} L \quad \text { and } \quad L_{R}=\frac{\hat{t}-t_{R}}{t_{U}-t_{R}} L
$$

After Hukou removal we need to add a migration equilibrium condition involving a money metric measure of the relative valuation of a unit of income across the two regions $\frac{T C L(U, \hat{t})}{T C L(R, \hat{t})}$, where TCL refers to the time cost of living index. This term reflects the different price levels across the two regions due to different housing prices. As TCL indices enter when making migration decisions, individuals take into account not only wage rate differences across regions, but also the differencet cost of housing.

To construct this metric we use the indirect utility functions

$$
\begin{array}{ll}
V_{t}\left(I_{t}^{U}\right)=\left\{\frac{1-t}{P_{G}}\right\}^{1-t}\left\{\frac{t}{P_{U}}\right\}^{t} I_{t}^{U}, & t \in\left[\hat{t}, t_{U}\right] \\
V_{t}\left(I_{t}^{R}\right)=\left\{\frac{1-t}{P_{G}}\right\}^{1-t}\left\{\frac{t}{P_{R}}\right\}^{t} I_{t}^{R}, & t \in\left[t_{R}, \hat{t}\right]
\end{array}
$$


for which the TCL indices are

$$
\begin{array}{ll}
T C L(U, t)=\left[\left\{\frac{1-t}{P_{G}}\right\}^{1-t}\left\{\frac{t}{P_{U}}\right\}^{t}\right]^{-1}, & t \in\left[\hat{t}, t_{U}\right] \\
T C L(R, t)=\left[\left\{\frac{1-t}{P_{G}}\right\}^{1-t}\left\{\frac{t}{P_{R}}\right\}^{t}\right]^{-1}, & t \in\left[t_{R}, \hat{t}\right]
\end{array}
$$

The equilibrium condition that migration must satisfy changes from the model with no house price effects and now becomes

$$
\frac{W_{U}}{W_{R}}=\frac{T C L(U, \hat{t})}{T C L(R, \hat{t})}=\left[\frac{P_{U}}{P_{R}}\right]^{\hat{t}}
$$

A general equilibrium after Hukou removal is thus given by the critical value of the share parameter $\hat{t}$, wage rates $W_{s}$, a goods price $P_{G}$, and urban and rural house prices $P_{U}$ and $P_{R}$ such that the following conditions hold.

[1] (Good Market Clearing) $\quad Y_{U}+Y_{R}=\int_{\hat{t}}^{t_{U}} G_{t}^{U} d t+\int_{t_{R}}^{\hat{t}} G_{t}^{R} d t$

[2] (House Market Clearing) $\quad \int_{\hat{t}}^{t_{U}} H_{t}^{U} d t=E_{U}$ and $\int_{t_{R}}^{\hat{t}} H_{t}^{R} d t=E_{R}$;

[3] (Labour Market Clearing) $\quad L_{U}+L_{R}=L$;

[4] (Migration Condition) $\quad \frac{W_{U}}{W_{R}}=\left[\frac{P_{U}}{P_{R}}\right]^{\hat{t}}$.

The general equilibrium conditions [2] can be written as

$$
\begin{gathered}
E_{U}=\int_{\hat{t}}^{t_{U}} H_{t}^{U} d t=\int_{\hat{t}}^{t_{U}} \frac{t}{P_{U}} I_{t}^{U} d t=\int_{\hat{t}}^{t_{U}} \frac{t}{P_{U}}\left(P_{G} Y_{U}+P_{U} E_{U}\right) X_{t}^{U} d t=\frac{P_{G} Y_{U}+P_{U} E_{U}}{P_{U}} \int_{\hat{t}}^{t_{U}} t X_{t}^{U} d t \\
P_{U} E_{U}=\frac{1}{2}\left(t_{U}+\hat{t}\right)\left(P_{G} Y_{U}+P_{U} E_{U}\right) \quad \text { and } \quad P_{U} E_{U}=\frac{\frac{1}{2}\left(t_{U}+\hat{t}\right)}{1-\frac{1}{2}\left(t_{U}+\hat{t}\right)} P_{G} Y_{U}
\end{gathered}
$$

and

$$
\begin{gathered}
E_{R}=\int_{t_{R}}^{\hat{t}} H_{t}^{R} d t=\int_{t_{R}}^{\hat{t}} \frac{t}{P_{R}} I_{t}^{R} d t=\int_{t_{R}}^{\hat{t}} \frac{t}{P_{R}}\left(P_{G} Y_{R}+P_{R} E_{R}\right) X_{t}^{R} d t=\frac{P_{G} Y_{R}+P_{R} E_{R}}{P_{R}} \int_{t_{R}}^{\hat{t}} t X_{t}^{R} d t \\
P_{R} E_{R}=\frac{1}{2}\left(\hat{t}+t_{R}\right)\left(P_{G} Y_{R}+P_{R} E_{R}\right) \quad \text { and } \quad P_{R} E_{R}=\frac{\frac{1}{2}\left(\hat{t}+t_{R}\right)}{1-\frac{1}{2}\left(\hat{t}+t_{R}\right)} P_{G} Y_{U}
\end{gathered}
$$

The general equilibrium condition [1] holds from equations (31) and (32).

To implement this model we again calibrate to base case data for 2001 and consider Hukou removal. ¿From Table 4 urban and rural non housing GDP in 2001 are 5849.777 and 4818.849. If we assume the shares of housing / apartments in GDP to be $22.50 \%$ and $18.50 \%,{ }^{7}$ then the base case value of the endowments of housing are 1316.199825 and 891.487065. The consumption value of non housing goods in the urban and rural areas is $4533.577175(=5849.777-1316.199825)$ and $3927.361935(=4818.849-891.487065)$. Urban and rural labour are 148.236 and 482.291 , and wage rates are 16.638050 and 5.739751. Rents are $2067.219177(=4533.577175-16.638050 \times 148.236)$ and $1159.131640(=3927.361935-5.739751 \times 482.291)$.

\footnotetext{
${ }^{7}$ See the discussion in Weng and Zuo (1999) which from a reading supports estimates of this size for the Mid 1990's appealing to survey evidence from various sources.
} 
Ignoring housing price effects, we can use the same simple model in Section 2 to analyze the effects of Hukou removal, but now the non housing portion of the economy is smaller. Through calibration to this smaller data we compute the scale and power parameters as $A_{U}=298.812076$ and $A_{R}=50.437145$, $\alpha_{U}=0.544020$ and $\alpha_{R}=0.704857$ through calibration. Equilibrium outcomes after Hukou removal are again in Table 7 - output: $Y_{U}=9116.089818$ and $Y_{R}=1251.721362$, work force: $L_{U}=535.295849$ and $L_{R}=95.231151$, common wage rate: $W_{U}=W_{R}=9.264667$. Labour migration under Hukou removal is $=535.295849-148.236=482.291-95.231151=387.059849$ and the efficiency gain to the economy from the elimination of Hukou restrictions is $=10367.811180-8460.939110=1906.872070$. 
Table 7. The Impacts of Hukou Removal in A General Equilibrium Model without House Prices

\begin{tabular}{|c|c|c|c|}
\hline \multicolumn{4}{|c|}{ A. Basic Data from Chinese Statistical Yearbook Used in Calibration } \\
\hline$Y_{U}^{0}=4533.577175$ & and & $Y_{R}^{0}=3927.361935$ & $Y^{0}=8460.939110$ \\
\hline$L_{U}^{0}=148.236$ & and & $L_{R}^{0}=482.291$ & $L^{0}=630.527$ \\
\hline \multicolumn{4}{|c|}{$W_{U}^{0}=16.638050$} \\
\hline \multicolumn{4}{|l|}{ B. Calibrated Model Parameters } \\
\hline \multicolumn{2}{|c|}{$A_{U}=298.812076$} & $A_{R}=$ & 50.437145 \\
\hline \multicolumn{2}{|c|}{$\alpha_{U}=0.544020$} & $\alpha_{R}=$ & .704857 \\
\hline \multicolumn{4}{|c|}{ C. Base Case Reference Equilibrium Before Hukou Removal } \\
\hline$Y_{U}=4533.577175$ & and & $Y_{R}=3927.361935$ & $Y=8460.939110$ \\
\hline$L_{U}=148.236$ & and & $L_{R}=482.291$ & $L=630.527$ \\
\hline \multicolumn{2}{|c|}{$W_{U}=16.638050$} & and & 5.739751 \\
\hline$I_{U}=4533.577175$ & and & $I_{R}=3927.361935$ & $I=8460.939110$ \\
\hline \multicolumn{4}{|c|}{ D. Counterfaction General Equilibrium After Hukou Removal } \\
\hline$Y_{U}=9116.089818$ & and & $Y_{R}=1251.721362$ & $Y=10367.811180$ \\
\hline \multirow[t]{2}{*}{$L_{U}=535.295849$} & and & $L_{R}=95.231151$ & $L=630.527000$ \\
\hline & $W_{U}$ & $=W_{R}=9.264667$ & \\
\hline$I_{U}=9116.089818$ & and & $I_{R}=1251.721362$ & $I=10367.811180$ \\
\hline Labour Migration $=$ & 35.295849 & $-148.236=482.29$ & $-95.231151=387.059849$ \\
\hline Gain $=$ & 10367.811 & $180-8460.939110=$ & 906.872070 \\
\hline
\end{tabular}


To evaluate the impacts of HUkou removal in the presence of house price effects, we use the model set out above in this Section. We assume the equilibrium prices in the base case are $P_{G}=1, P_{U}=1$ and $P_{R}=1$. $Y_{U}=4533.577175$ and $Y_{R}=3927.361935, E_{U}=1316.199825$ and $E_{R}=891.487065, L_{U}=148.236$ and $L_{R}=482.291, W_{U}=16.638050$ and $W_{R}=5.739751, R_{U}=2067.219177$ and $R_{R}=1159.131640$, $I_{U}=4533.577175$ and $I_{R}=3927.361935$. Through calibration we compute the scale and power parameters as $A_{U}=298.812076$ and $A_{R}=50.437145, \alpha_{U}=0.544020$ and $\alpha_{R}=0.704857$.

If in this case we also assume $t_{U}=0.234404$ and $t_{R}=0.154404$, this implies $\hat{t}=0.215596$. Thus $\int_{\hat{t}}^{t_{U}} I_{t}^{U} d t=5849.777$ and $\int_{t_{R}}^{\hat{t}} I_{t}^{R} d t=4818.849$. Then $\int_{\hat{t}}^{t_{U}} G_{t}^{U} d t=4533.577175$ and $\int_{\hat{t}}^{t_{U}} H_{t}^{U} d t=1316.199825$, $\int_{t_{R}}^{\hat{t}} G_{t}^{R} d t=3927.361935$ and $\int_{t_{R}}^{\hat{t}} H_{t}^{R} d t=891.487065$.

Table 8 presents results of Hukou removal in this case. We assume that in the base case $t_{U}=0.234404$ and $t_{R}=0.154404$, then this implies $\hat{t}=0.176035$. The equilibrium prices are $P_{G}=1.000000, P_{U}=1.646876$ and $P_{R}=0.418946$. Other variables are $Y_{U}=8394.802167$ and $Y_{R}=1887.050537, L_{U}=460.035079$ and $L_{R}=170.491921, W_{U}=9.927379$ and $W_{R}=7.801552, R_{U}=3827.859405$ and $R_{R}=556.948919$, $I_{U}=8394.802167$ and $I_{R}=1887.050537, \int_{\hat{t}}^{t_{U}} I_{t}^{U} d t=10562.419584$ and $\int_{t_{R}}^{\hat{t}} I_{t}^{R} d t=2260.535756$. Under Hukou elimination $\int_{\hat{t}}^{t_{U}} G_{t}^{U} d t=8394.802167$ and $\int_{\hat{t}}^{t_{U}} H_{t}^{U} d t=2167.617417, \int_{t_{R}}^{\hat{t}} G_{t}^{R} d t=1887.050537$ and $\int_{t_{R}}^{\hat{t}} H_{t}^{R} d t=373.485219$. Labour migration under Hukou removal is $=460.035079-148.236=482.291-$ $170.491921=311.799079$ and the efficiency gain from elimination of Hukou restrictions is $=12822.955340-$ $10668.626=2154.329340$. Rising house prices thus serve to dampen migration and reduce the size of the efficiency gain. 
Table 8. The Impacts of Hukou Removal in A General Equilibrium Model with House Prices

A. Basic Data from Chinese Statistical Yearbook Used in Calibration

$$
\begin{aligned}
& Y_{U}^{0}=4533.577175 \quad \text { and } \quad Y_{R}^{0}=3927.361935 \quad Y^{0}=8460.939110 \\
& L_{U}^{0}=148.236 \quad \text { and } \quad L_{R}^{0}=482.291 \quad L^{0}=630.527 \\
& W_{U}^{0}=16.638050 \quad \text { and } \quad W_{R}^{0}=5.739751 \\
& E_{U}=1316.199825 \quad \text { and } \quad E_{R}=891.487065
\end{aligned}
$$

B. Calibrated Model Parameters

$$
\begin{array}{rll}
A_{U}=298.812076 & \text { and } & A_{R}=50.437145 \\
\alpha_{U}=0.544020 & \text { and } & \alpha_{R}=0.704857
\end{array}
$$

C. Base Case Reference Equilibrium Before Hukou Removal

$$
\begin{aligned}
& t_{U}=0.234404 \quad \text { and } \quad t_{R}=0.154404 \quad \hat{t}=0.215596 \\
& P_{G}=1.00000 \quad P_{U}=1.00000 \quad \text { and } \quad P_{R}=1.00000 \\
& Y_{U}=4533.577175 \quad \text { and } \quad Y_{R}=3927.361935 \quad Y=8460.939110 \\
& L_{U}=148.236 \quad \text { and } \quad L_{R}=482.291 \quad L=630.527 \\
& W_{U}=16.638050 \quad \text { and } \quad W_{R}=5.739751 \\
& I_{U}=4533.577175 \quad \text { and } \quad I_{R}=3927.361935 \quad I=8460.939110 \\
& \int_{\hat{t}}^{t_{U}} I_{t}^{U} d t=5849.777 \quad \text { and } \quad \int_{t_{R}}^{\hat{t}} I_{t}^{R} d t=4818.849 \quad \int_{t_{R}}^{\hat{t}}\left(I_{t}^{U}+I_{t}^{R}\right) d t=10668.626 \\
& \int_{\hat{t}}^{t_{U}} G_{t}^{U} d t=4533.577175 \quad \text { and } \quad \int_{\hat{t}}^{t_{U}} H_{t}^{U} d t=1316.199825 \\
& \int_{t_{R}}^{\hat{t}} G_{t}^{R} d t=3927.361935 \quad \text { and } \quad \int_{t_{R}}^{\hat{t}} H_{t}^{R} d t=891.487065
\end{aligned}
$$

D. Counterfaction General Equilibrium After Hukou Removal

$$
\begin{aligned}
& t_{U}=0.234404 \quad \text { and } \quad t_{R}=0.154404 \quad \hat{t}=0.176036 \\
& P_{G}=1.000000 \quad P_{U}=1.646876 \quad \text { and } \quad P_{R}=0.418946 \\
& Y_{U}=8394.802167 \quad \text { and } \quad Y_{R}=1887.050537 \quad Y=10281.852705 \\
& L_{U}=460.035079 \quad \text { and } \quad L_{R}=170.491921 \quad L=630.527000 \\
& W_{U}=9.927379 \quad \text { and } \quad W_{R}=7.801552 \\
& I_{U}=8394.802167 \quad \text { and } \quad I_{R}=1887.050537 \quad I=10281.852705 \\
& \int_{\hat{t}}^{t_{U}} I_{t}^{U} d t=10562.419584 \quad \text { and } \quad \int_{t_{R}}^{\hat{t}} I_{t}^{R} d t=2260.535756 \quad \int_{t_{R}}^{\hat{t}}\left(I_{t}^{U}+I_{t}^{R}\right) d t=12822.955340 \\
& \int_{\hat{t}}^{t_{U}} G_{t}^{U} d t=8394.802167 \quad \text { and } \quad \int_{\hat{t}}^{t_{U}} H_{t}^{U} d t=2167.617417 \\
& \int_{t_{R}}^{\hat{t}} G_{t}^{R} d t=1887.050537 \quad \text { and } \quad \int_{t_{R}}^{\hat{t}} H_{t}^{R} d t=373.485219
\end{aligned}
$$

Labour Migration $=460.035079-148.236=482.291-170.491921=311.799079$

Gain $=10281.852705-8460.939110=1820.913595$ 


\section{CONCLUSIONS AND REMARKS}

This paper studies the impacts of the Hukou system of permanent registration on income inequality and labour migration in China. Its aim is to use numerical modelling to help assess the contribution of various policy and other factors in China in either contributing to or retarding inequality in China. We use three model variants to develop results. The base model is taken from Hamilton and Whalley (1984), who evaluate the impacts of cross country immigration restrictions on global inequality and examines four 2 region cases, a 3 region case, a 6 region case, and 31 province case in which alternative and groupings divisions of Chinese data are used. Our extension allows for productivity differences across individuals within each region in the model and explores income inequality impacts for five model cases. A second house model captures the effects of higher urban house prices in retarding rural labour movement in to urban areas.

All model results point towards a significant role of the Hukou system in preventing movement towards a more equal distribution of income in China. There effects are smaller in the second two model variants than in the first. We see all models as a simplification from a more complex reality, and so we do not aim to provide from point estimate of impact. But the themes of results seem clear, and in addition we offer new methodological approaches which can also be used for the analysis of mobility restrictions other economies. 


\section{REFERENCES}

[1] Chris Bramall and Marion E.Jones (1993) Rural Income Inequality in China since 1978. Journal of Peasant Studies, 21, 41-70

[2] Kam Wing Chan and Li Zhang (1999) The Hukou System and Rural - Urban Migration in China: Processes and Changes. China Quarterly, 160, December 1999, 818-855

[3] Gene H Chang (2002) The Cause and Cure of China's Widening Income Disparity. Department of Economics, University of Toledo http://unr.edu/homepage/elliottp/cer134chang.pdf

[4] Jian Chen (1996) Regional Income Inequality and Economic Growth in China. Journal of Comparative Economics, 22, 141-164

[5] China State Statistics Bureau (2002) China Statistical Yearbook. Beijing: China Statistical Press

[6] Xiaoyu Fan and Shufen Qie (2002) The Situation and Features of Rural Labour Migration. China's Statistics, 7 (in Chinese)

[7] Denise Hare and Loraine A.West (1999) Spatial Patterns in China's Rural Industrial Growth and Prospects for the Alleviation of Regional Income Inequality. Journal of Comparative Economics, 27, $475-497$

[8] Bob Hamilton and John Whalley (1984) Efficiency and Distributional Implications of Global Restrictions on Labour Mobility. Journal of Development Economics, 14, 61-75

[9] Jyotsna Jalan and Martin Ravallion (1998) Transient Poverty in Postreform Rural China, 1952-1985. Journal of Comparative Economics, 26, 338-357

[10] Ravi Kanbur and Xiaobo Zhang (1999) Which Regional Inequality? The Evolution of Rural Urban and Inland - Coastal Inequality in China from 1983 to 1995. Journal of Comparative Economics, 27, 686-701

[11] Ravi Kanbur and Xiaobo Zhang (2001)

Fifty Years of Regional Inequality in China: A Journey through Revolution, Reform and Openness. Working Paper 2001 - 04, Department of Economics and Management, Cornell University

[12] Aying Liu, Shujie Yao and Zongyi Zhang (2001) Convergence of China's Regional Income: 1952 97. Chinese Economics Review, 12, 243-358

[13] Thomas P.Lyons (1991) Interprovincial Disparities in China: Output and Consumption, 1952-1987. Economic Development Cultural Change, 39, 471-506

[14] Scott Rozelle (1994) Rural Industrialization and Increasing Inequality: Emerging Patterns in China's Reforming Economy. Journal of Comparative Economics, 19, 362-391

[15] Amartya Sen (1973) On Economic Inequality. Oxford University Press

[16] Xinzheng Shi (2002) Empirical Research on Urban - Rural Income Differentials: The Case of China. Unpublished Manuscript, CCER, Peking University

[17] Xinzheng Shi, Terry Sicular and Yaohui Zhao (2002) Analyzing Urban - Rural Income Inequality in China. Paper presented at the International Symposium on Equality and Social Justice in Transitional China, Beijing, July 11 - 12

[18] Kai-yuen Tsui (1991) China's Regional Inequality, 1952-1985. Journal of Comparative Economics, 
15, 1-21

[19] Kai-yuen Tsui (1993) Decomposition of China's Regional Inequalities. Journal of Comparative Economics, 17, 600-627

[20] Kai-yuen Tsui (1996) Economic Reform and Interprovincial Inequality in China. Journal of Development Economics, 50, 353-368

[21] Kai-yuen Tsui (1998) Factor Decomposition of Chinese Rural Income Inequality: New Methodology, Empirical Findings, and Policy Implications. Journal of Comparative Economics, 26, 502-528

[22] Kai-yuen Tsui (1998) Trends and Inequalities of Rural Welfare in China: Evidence from Rural Households in Guangdong and Sichuan. Journal of Comparative Economics, 26, 783-804

[23] Feng Wang and Xuejin Zuo (1999) Inside China's Cities: Institutional Barriers and Opportunities for Urban Migrants. American Economic Review: AEA Papers and Proceedings, 89 (2), 276-280

[24] Fan Zhan and Zhi Wang (2002) WTO Accession, Rural Labour Migration and Urban Unemployment in China. $\quad$ Urban Studies, 39 (12), 2199 - 2217

[25] Yaohui Zhao (1999) Labour Migration and Earning Differences: The Case of Rural China. Economic Development and Culture Change, 47 (4), 767 - 782

[26] Yaohui Zhao (1999) Leaving the Countryside: Rural - to - Urban Migration Decisions in China. American Economic Review, 89, 281 - 286 